\title{
Do injection drug users have more adverse events during procedural sedation and analgesia for incision and drainage of cutaneous abscesses?
}

Frank Xavier Scheuermeyer, MD, MHSc*; Gary Andolfatto, $\mathrm{MD}^{\dagger}$; Lisa Lange, MD*; Danielle de Jong, $\mathrm{MD}^{*}$; Hong Qian, $\mathrm{PhD}^{\ddagger}$; Eric Grafstein, $\mathrm{MD}^{\S}$

ABSTRACT

Objective: Injection drug users (IDUs) often undergo procedural sedation and analgesia (PSA) in the emergency department (ED). We compared adverse events (AEs) for IDUs to those for non-IDUs receiving PSA for incision and drainage of cutaneous abscesses.

Methods: This was a retrospective analysis of a PSA safety audit. IDU status was prospectively documented among consecutive patients undergoing PSA at two urban EDs. Structured data describing comorbidities, vital signs, sedation regimens, and adverse events were collected. Primary outcome was the proportion of patients in each group experiencing an $A E$, whereas the secondary outcomes included recovery times.

Results: Of 525 consecutive patients receiving PSA for incision and drainage of an abscess, 244 were deemed IDUs and 281 non-IDUs. IDUs received higher doses of sedatives and analgesics, and 14 experienced AEs (5.7\%), whereas 10 non-IDUs had AEs (3.6\%), for a risk difference of $2.1 \%(95 \% \mathrm{Cl}-1.8,6.5)$. Median recovery times were 18 minutes (interquartile range [IOR] 10-36) for IDUs and 12 minutes (IQR 7-19) for non-IDUs, for a difference of 6 minutes $(95 \% \mathrm{Cl} 2-9$ minutes). Median sedation times were also longer in IDUs, for a difference of 6 minutes $(95 \% \mathrm{Cl} 5-10$ minutes). Of 20 IDU patients and 1 non-IDU patient admitted to hospital, none had experienced an AE related to PSA. Conclusions: For ED patients requiring PSA for incision and drainage, IDUs had an AE rate similar to that of non-IDUs but longer sedation and recovery times. In experienced hands, PSA may be as safe in IDUs as in patients who do not use injection drugs.
RÉSUMÉ

Objectif: Les utilisateurs de drogues injectables (UDI) subissent souvent des interventions sous sédation-analgésie (SA) aux services des urgences (SU). L'étude visait à comparer les événements indésirables (EI) de la SA chez les UDI avec ceux observés chez les non-UDI, pour l'incision et le drainage d'abcès cutanés.

Méthode: II s'agit d'une analyse rétrospective d'une vérification sur l'innocuité de la SA. La situation d'UDI a été établie de manière prospective parmi des patients consécutifs ayant subi une SA dans deux SU urbains, et il y a eu collecte de données structurées sur les affections concomitantes, les signes vitaux, la posologie des sédatifs administrés, et les événements indésirables. Le principal critère d'évaluation était la proportion de patients dans chaque groupe qui avait éprouvé des $\mathrm{El}$, et les critères d'évaluation secondaires comprenaient, entre autres, le temps de réveil.

Résultats: Sur 525 patients consécutifs, ayant subi une SA pour l'incision et le drainage d'un abcès, 244 étaient considérés comme des UDI et 281, comme des non-UDI. Les UDI ont reçu des doses plus fortes de sédatifs et d'analgésiques, et 14 ont éprouvé des EI (5.7\%), tandis que 10 non-UDI ont connu des El (3.6\%), soit une différence de risques de 2.1 $\%$ (IC à $95 \%-1.8,6.5$ ). Pour ce qui est du temps médian de réveil, celui-ci était de 18 minutes (intervalle interquartile [II]: 10-36) chez les UDI contre 12 minutes (II: 7-19) chez les nonUDI, soit un écart de 6 minutes (IC à $95 \%$ 2-9 minutes). Quant au temps médian de sédation, celui-ci était également plus long chez les UDI que chez les non-UDI, soit un écart de 6 minutes (IC à 95\% 5-10 minutes). Sur 20 UDI et 1 non-UDI hospitalisés, aucun n'a éprouvé d'El lié à la SA.

From the *Department of Emergency Medicine, St Paul's Hospital and the University of British Columbia, Vancouver, BC; †Department of Emergency Medicine, Lions Gate Hospital and the University of British Columbia, North Vancouver, BC; $¥$ Center for Health Evaluation and Outcome Sources (CHEOS), St Paul's Hospital and the University of British Columbia, Vancouver, BC; and §Department of Emergency Medicine, Mount St Joseph's Hospital and the University of British Columbia, Vancouver, BC.

Correspondence to: Dr. Frank Scheuermeyer, Department of Emergency Medicine, St Paul's Hospital, 1081 Burrard Street, Vancouver BC; frank. scheuermeyer@gmail.com.

This article has been peer reviewed. 
Conclusions: En ce qui concerne les patients traités aux SU et ayant subi une SA pour incision et drainage, les UDI avaient un taux d'El comparable à celui des non-UDI; par contre, les temps de sédation et de réveil étaient plus longs chez les premiers que chez les seconds. Confiée à des praticiens chevronnés, la SA effectuée chez les UDI peut être aussi sûre que celle effectuée chez les non-UDI.

Keywords: infectious diseases, marginalized populations, pain management
Injection drug users (IDUs), an estimated 13 million persons worldwide, are predisposed to a variety of serious medical sequelae. ${ }^{1}$ The emergency department (ED) is a common venue for treatment due to the often chaotic social circumstances of IDUs. ${ }^{2,3}$ For example, the incision and drainage of cutaneous abscesses under procedural sedation and analgesia (PSA) is a typical ED procedure in hospitals serving a large population of IDUs.

IDUs are thought to have altered responses for both analgesics and sedatives compared to nonIDUs. ${ }^{4-6}$ Long-standing substance misuse may sensitize patients to various anesthetic agents. ${ }^{7,8}$ Peripheral intravenous access may be difficult ${ }^{9}$ and pain tolerance may be decreased, requiring altered doses of analgesia..$^{10,11}$

We compared dosing of sedative and analgesic medications, adverse event (AE) rates, and recovery times in IDUs and non-IDUs undergoing PSA for incision and drainage of cutaneous abscesses.

\section{METHODS}

\section{Study design}

This was a retrospective analysis of data that were prospectively collected for a regional safety audit on all patients undergoing PSA.

\section{Study setting and population}

The study was conducted at two urban sites in the Vancouver Coastal Health region and covered ED encounters from April 1, 2006, to January 31, 2009. St Paul's Hospital (site 1) is a tertiary care centre with an annual census of 60,000 ED visits, and Mount Saint Joseph's Hospital (site 2) is a community hospital with 30,000 ED visits per year. Both institutions are teaching centres affiliated with the University of British Columbia and are staffed by full-time emergency physicians, approximately one-quarter of whom work at both sites. Data collection was approved by the ethics committees of both institutions.

The two sites share an administrative database that captures all patient demographics, including mode of arrival, age, gender, address, chief complaint, and times of arrival and discharge. Hospital policy specifies that emergency physicians complete an electronic chart summary on all patients-composed of a list of all diagnoses and ED procedures-which is linked to the database. This database was interrogated by searching for the procedure codes of "procedural sedation" and "incision and drainage." We selected incision and drainage as this is a common procedure performed on both IDUs and non-IDUs. A previous similar audit of "procedural sedation" and "electrical cardioversion" demonstrated a physician coding accuracy of $99 \% .{ }^{12}$

At both ED sites, each PSA was performed by the attending emergency physician. As per regional policy, physicians were assisted by registered nurses and respiratory therapists who were specially trained in ED PSA, and cardiac, noninvasive blood pressure, and pulse oximetry monitoring was mandatory. The attending physician alone selected the sedation regimen, including presedation opioids. The same physician performed the incision and drainage when the patient was deemed to have reached a sufficiently dissociated state or deep level of sedation. ${ }^{13}$ Head, neck, trunk, and extremity abscesses were typically drained with patients in the supine position, whereas buttock, perianal, and ischiorectal abscesses were typically drained in the lateral decubitus position. Physicians individualized the use of local or regional anesthesia, postsedation analgesia, and follow-up.

\section{Data collection}

As part of a regional quality assurance program, all patients undergoing PSA had information recorded at the time of sedation on standardized data collection forms. The attending physician or nurse recorded patient comorbidities, IDU status (see below), body weight, American Society of Anesthesiologists' (ASA) risk score, and the last solid and liquid intake. All sedative and analgesic medications administered were 
recorded, along with dose and time. Vital signs were documented before the procedure, whenever a medication dose was administered, whenever an adverse event was deemed to have occurred by the treating physician, and every 5 minutes until physiologic recovery. AEs were documented according to regional guidelines by the attending physician or nurse on the data collection sheet and are described below under "Outcome measures."

As part of regional policy, the attending physician or registered nurse asked patients, prior to undergoing PSA, a standardized question: "Have you used injection drugs in the past month?" A similar approach has been used to identify IDUs entering the Vancouver Injection Drug Use Study since 1996. ${ }^{14-16}$ Although self-reported illicit drug use appears to be accurate, ${ }^{17}$ physicians and nurses used supporting evidence such as a previous history of injection drug use, recent abscesses, track marks, and clinical illicit drug intoxication to confirm or refute the patient's answer.

After the procedure, recovery was continuously supervised by the nurse and respiratory therapist, who recorded patient vital signs and activity every 5 minutes using a modified Aldrete scale. ${ }^{18}$

\section{Data abstraction}

We followed standards for medical record review, except that data abstractors were not blinded to study hypothesis. ${ }^{19,20}$ Three trained emergency physician reviewers scrutinized the standardized data sheets and accompanying patient charts and recorded information directly into a specially prepared Microsoft Excel 2008 (Microsoft Corp, Redmond, WA) database. IDU status was recorded from the checkbox on the data sheet. When the status was not recorded or was incongruent with the clinical presentation (e.g., a patient who denied injection drug use but had a new antecubital fossa abscess), the electronic chart was reviewed to 1999-the start of electronic charting at our institutions-to ascertain status. If the status was still unclear, the case was adjudicated independently by an addictions medicine specialist and an infectious diseases specialist who were blinded to all other information. If they could not agree, the primary investigator determined the status.

All PSA medications administered, along with dose and time and vital signs at the start and finish of the procedure, were recorded. Any AEs noted by the treating physician were abstracted, along with vital signs and interventions.

Missing data sheet information was obtained when possible by reviewing the patient chart to 1999 (the start of electronic charting at our institutions). A random $10 \%$ of charts were independently abstracted by a second reviewer, and interobserver reliability scores calculated for IDU status and ambulance arrival. All patients having an AE were reviewed by a second emergency physician reviewer to ascertain whether an AE had taken place. Disputed, missing, and unclear chart information was resolved by consensus at regularly planned review meetings.

\section{Outcome measures}

The primary outcome was the proportion of patients having an $\mathrm{AE}$ in each group. AEs were defined by Vancouver Coastal Health regional PSA guidelines ${ }^{21-23}$ and are explained in Table 1. AEs included airway obstruction, apnea, hypoxia; hypotension, recovery agitation (dysphoric behaviour, shouting, or agitation during or after recovery), myoclonus, and nausea or emesis. Other AEs included use of reversal agents, recovery time $>3$ hours, and PSA-related hospital admissions. A patient could have more than one AE;

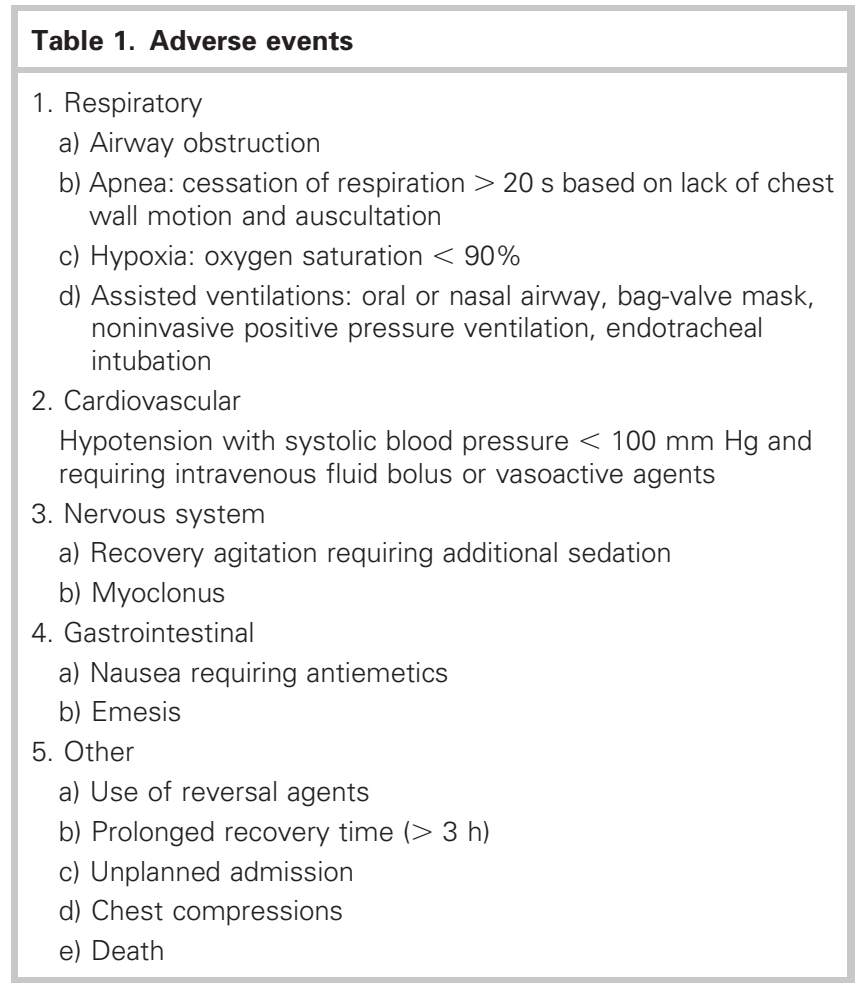


for example, an apneic patient who was nauseated might receive an oral airway and an antiemetic.

Recovery time was measured by the Aldrete scale. ${ }^{18}$ Once a patient reached a score of 8 of 10 , he or she was deemed "recovered" from PSA. Secondary outcomes included recovery time (i.e., the difference between the administration of the last PSA medication and recovery), sedation time (i.e., difference between administration of the first PSA medication and recovery), and hospital admission.

\section{Data analysis}

Data are reported in terms of proportions or means and standard deviations; nonparametric data are summarized as medians and interquartile ranges (IQRs). A bootstrap method in $R$ (version 2.13.1) was used to estimate confidence intervals for differences between medians. Scatterplots were generated with Excel 2008, and boxplots were generated using the default "boxplot" command in $R$.

\section{RESULTS}

Data were collected on 525 consecutive patients, of whom 244 were IDUs and 281 were non-IDUs. Patient weight was missing in 9\% of cases and ASA classifications in $11 \%$. Kappa values were 0.88 for IDU status and 0.90 for ambulance arrival. Nine patients required adjudication to determine IDU status. Table 2 compares presedation characteristics between the two groups. IDUs had higher rates of abnormal vital signs at baseline.

Table 3 classifies the sedation regimens as propofol alone, propofol/fentanyl, fentanyl/midazolam, and ketamine/propofol. IDUs received higher doses for all sedatives and analgesics, but significant differences were observed only for propofol dosing in the propofol-alone group and for fentanyl dosing in the fentanyl/midazolam group. Similar proportions of patients received preprocedural opioid analgesia.

AEs are summarized in Table 4. Among IDUs, 14 of 244 patients had AEs (5.7\%, 95\% CI 3.3-9.7), whereas 10 of 281 non-IDUs had AEs (3.6\%, 95\% CI 1.8-6.6), for a risk difference of $2.2 \%(95 \%$ CI $-1.8,6.5)$ No patient died or received chest compressions or endotracheal intubation. IDUs had greater rates of hypotension $(1.2 \%$ versus $0.0 \%)$, recovery agitation $(0.82 \%$ versus 0.0$)$, and prolonged recovery time $>3$ hours $(1.2 \%$ versus $0.36 \%)$, but these differences were not statistically significant.

Table 5 details the 24 patients with AEs. Figure 1 displays the relationship between the cumulative dose of medication administered and AEs for each of the twodrug PSA regimens. No patient given propofol alone developed an AE. The majority of patients (13 of 24) with AEs had respiratory events, and 9 patients received reversal agents. Overall, 144 of 244 (59.0\%) IDUs were opioid dependent and 8 of 14 IDUs (57.1\%) with AEs used heroin. The 14 IDUs with AEs had a median recovery time of 59 minutes (IQR 35-182 minutes), whereas the 10 non-IDUs with AEs had a recovery time of 23 minutes (IQR 11-46 minutes).

Secondary outcomes are described in Table 6 and illustrated in Figure 2. Both recovery times and sedation times were on average 6 minutes longer in IDUs. Twenty IDUs and one non-IDU (who was homeless and used cocaine) were admitted to hospital. None of these 21 admitted patients had experienced an AE related to PSA.

\section{DISCUSSION}

In a consecutive series of patients receiving PSA for incision and drainage of cutaneous abscesses, IDUs had a $5.7 \% \mathrm{AE}$ rate, whereas non-IDUs had a 3.6\% AE rate, for a nonsignificant risk difference of $2.1 \%$. Importantly, all AEs resolved with minimal interventions, and no patients died or had an unplanned admission related to sedation. IDUs were administered higher doses of sedative and analgesic medications than their non-IDU counterparts. Both recovery and sedation times were longer in the IDU group.

In theory, IDUs have a higher potential for sedation-related adverse events. ${ }^{48}$ Chronic liver disease, poor nutrition, and decreased intravascular volume can lead to altered pharmacokinetics of sedatives and analgesics. Pre-ED use of opioids can predispose patients to respiratory depression during PSA, especially when combined with further sedatives. When used during PSA, ketamine increases heart rate and blood pressure and may potentiate the cardiac effects of cocaine. ${ }^{24}$ Patients who misuse cocaine undergo pronounced changes in plasma endorphin levels and alterations in opioid mu and kappa receptors, ${ }^{4,5}, 7$ possibly resulting in abnormal pain perception and higher requirements for analgesics. ${ }^{6}$ These features may in part account for higher 


\begin{tabular}{|c|c|c|}
\hline Category & $\operatorname{IDU}(n=244)$ & Non-IDU $(n=281)$ \\
\hline Age, mean (SD) & $36.5(9.4)$ & $40.8(12.0)$ \\
\hline Gender, $n$ (\% male) & $146(59.9)$ & 219 (77.9) \\
\hline Arrival by ambulance, $n(\%)$ & $38(15.5)$ & $8(2.9)$ \\
\hline Site 1 & $244(90.1)$ & $212(75.4)$ \\
\hline Site 2 & $20(9.9)$ & $69(24.6)$ \\
\hline \multicolumn{3}{|l|}{ Vital signs at the start of procedures, mean (SD) } \\
\hline Systolic blood pressure in $\mathrm{mm} \mathrm{Hg}$ & $120.4(16.3)$ & $126.9(18.0)$ \\
\hline Diastolic blood pressure in $\mathrm{mm} \mathrm{Hg}$ & $72.3(11.1)$ & $75.1(10.6)$ \\
\hline Heart rate in beats per minute & $94.9(15.9)$ & $90.1(16.2)$ \\
\hline Respiratory rate in breaths per minute & $18.9(1.9)$ & $18.2(2.0)$ \\
\hline Oxygen saturation in \% & $97.7(1.5)$ & $97.7(1.5)$ \\
\hline Temperature in degrees Celsius & $37.1(0.7)$ & $36.9(0.6)$ \\
\hline \multicolumn{3}{|l|}{ Patients with abnormal vital signs at the start of procedures, $n(\%)$} \\
\hline Systolic blood pressure $<100 \mathrm{~mm} \mathrm{Hg}$ & $27(11.1)$ & $18(6.4)$ \\
\hline Diastolic blood pressure $<60 \mathrm{~mm} \mathrm{Hg}$ & $33(13.5)$ & $17(6.0)$ \\
\hline Heart rate $>100$ beats per minute & 96 (39.3) & $63(22.4)$ \\
\hline Temperature $>37.5^{\circ} \mathrm{C}$ & $54(22.1)$ & $27(9.6)$ \\
\hline \multicolumn{3}{|l|}{ Drugs of abuse, $n(\%)^{*}$} \\
\hline Heroin & $144(59.0)$ & $0(0.0)$ \\
\hline Cocaine & $89(36.4)$ & $47(16.7)$ \\
\hline Amphetamines & 29 (11.9) & $11(3.9)$ \\
\hline Alcohol & $10(4.1)$ & $12(4.1)$ \\
\hline \multicolumn{3}{|l|}{ Comorbidities, $n(\%)$} \\
\hline HIV & $43(17.7)$ & $30(10.7)$ \\
\hline Hepatitis C & $134(54.9)$ & $42(14.9)$ \\
\hline \multicolumn{3}{|l|}{ ASA class, $n(\%)$} \\
\hline 1 & $151(61.9)$ & $177(63.0)$ \\
\hline 2 & $64(26.2)$ & $67(23.8)$ \\
\hline 3 & $1(0.4)$ & $6(2.1)$ \\
\hline 4 or 5 & $0(0.0)$ & $0(0.0)$ \\
\hline Not recorded & $28(11.5)$ & $31(11.0)$ \\
\hline ED opioid analgesia given $<30$ min prior to PSA, $n(\%)$ & $65(26.7)$ & $78(27.8)$ \\
\hline Morphine & $58(23.8)$ & $49(17.4)$ \\
\hline Dose morphine, mg/kg, median (IQR) & $0.07(0.04-0.12)$ & $0.10(0.07-0.14)$ \\
\hline Fentanyl & $7(2.9)$ & $29(10.4)$ \\
\hline Dose fentanyl, $\mu \mathrm{g} / \mathrm{kg}$, median (IQR) & $0.71(0.58-0.89)$ & $0.65(0.54-0.72)$ \\
\hline \multicolumn{3}{|l|}{ Abscess location, $n(\%)$} \\
\hline Trunk (abdomen/chest/back) & $15(6.2)$ & $34(12.1)$ \\
\hline Head/neck & $13(5.3)$ & $11(3.9)$ \\
\hline Buttocks/perianal/ischiorectal & $33(13.5)$ & $130(46.3)$ \\
\hline Extremities & $183(75.0)$ & $106(37.7)$ \\
\hline
\end{tabular}

cumulative doses of sedative and analgesics in the IDU group.

Despite these higher doses, the overall $\mathrm{AE}$ rate was comparable in both groups. Several factors may explain this result. Our patients may have received lower doses of sedatives and analgesics relative to their needs. All of the IDUs and some of the non-IDU group had substance misuse issues, and applying conventional dosing to this population may result in oligoanalgesia. Incision and drainage, which involves infiltration of an abscess with local anesthetic, incising the abscess, exploring and unroofing the abscess, and packing the resulting cavity, is a long, exquisitely painful procedure. It is possible that the noxious stimulation 


\begin{tabular}{|c|c|c|c|}
\hline Category & $\operatorname{IDU}(n=244)$ & Non-IDU $(n=281)$ & $\%$ Difference $(95 \% \mathrm{Cl})^{*}$ \\
\hline \multicolumn{4}{|c|}{ Sedation regimens, $n(\%)$} \\
\hline Propofol & $64(26.2)$ & $94(33.4)$ & $-7.2(-15.0,0.92)$ \\
\hline Propofol/fentanyl & $82(33.6)$ & $72(25.6)$ & $8.0(0.11,16.0)$ \\
\hline Fentanyl/midazolam & $56(23.0)$ & $60(21.4)$ & $1.6(-5.7,9.0)$ \\
\hline Ketamine/propofol & $42(17.2)$ & 55 (19.6) & $-2.4(-9.2,4.6)$ \\
\hline \multicolumn{4}{|c|}{ Sedation regimen doses, median (IQR) } \\
\hline Propofol (mg/kg) & $2.22(1.51-2.99)$ & $1.82(1.33-2.26)$ & $0.40(0.26,0.61)$ \\
\hline Propofol (mg/kg) & $2.11(1.47-2.86)$ & $1.96(1.14-2.43)$ & $0.15(-0.10,0.31)$ \\
\hline Fentanyl $(\mu \mathrm{g} / \mathrm{kg})$ & $1.31(0.83-1.67)$ & $1.23(0.86-1.69)$ & $0.08(-0.19,0.28)$ \\
\hline Fentanyl $(\mu \mathrm{g} / \mathrm{kg})$ & $3.10(2.50-5.00)$ & $2.44(1.77-3.00)$ & $0.66(0.22,1.25)$ \\
\hline Midazolam (mg/kg) & $0.05(0.03-0.08)$ & $0.04(0.02-0.05)$ & $0.01(-0.01,0.02)$ \\
\hline Ketamine $(\mathrm{mg} / \mathrm{kg})$ & $0.80(0.47-1.00)$ & $0.63(0.44-0.84)$ & $0.17(-0.11,0.40)$ \\
\hline Propofol (mg/kg) & $1.20(0.87-1.54)$ & $0.94(0.58-1.20)$ & $0.26(-0.08,0.50)$ \\
\hline
\end{tabular}

provided by the procedure may have stimulated patients sufficiently to prevent hypotension or adverse respiratory events. Interestingly, IDUs who abused opioids did not appear to have a higher proportion of AEs than IDUs not abusing opioids.
As displayed in the scatterplots, higher doses of sedative and analgesics appeared to correlate with more AEs. More specifically, many AEs occurred when patients received beyond the 75 th percentile dose for at least one of the medications, especially fentanyl when

\begin{tabular}{|c|c|c|c|}
\hline Category & $\begin{array}{l}\text { IDU }(n=244) \\
n(\%, 95 \% \mathrm{CI})\end{array}$ & $\begin{array}{c}\text { Non-IDU }(n=281) \\
n(\%, 95 \% \mathrm{Cl})\end{array}$ & $\begin{array}{l}\% \text { Difference } \\
(95 \% \mathrm{Cl})^{\dagger}\end{array}$ \\
\hline \multicolumn{4}{|l|}{ Adverse events } \\
\hline Total number of patients with an $\mathrm{AE}$ & $14(5.7,3.3-9.7)$ & $10(3.6,1.0-6.6)$ & $2.2(-1.8,6.5)$ \\
\hline Total number of AEs & $18(7.4,4.6-11.6)$ & $15(5.3,3.2-8.8)$ & $2.0(-2.5,6.8)$ \\
\hline \multicolumn{4}{|l|}{ Respiratory } \\
\hline Airway obstruction & $0(0.0,0.0-1.2)$ & $2(0.71,0.002-2.7)$ & $-0.71(-2.8,1.3)$ \\
\hline Apnea & $2(0.82,0.03-3.1)$ & $3(1.1,0.02-3.2)$ & $-0.28(-2.3,2.6$ \\
\hline Hypoxia & $3(1.2,0.03-3.7)$ & $3(1.1,0.02-3.2)$ & $0.10(-1.7,3.5)$ \\
\hline \multicolumn{4}{|l|}{ Cardiovascular } \\
\hline Hypotension & $3(1.2,0.03-3.7)$ & $0(0.0,0.0-1.1)$ & $1.2(-0.7,3.9)$ \\
\hline \multicolumn{4}{|l|}{ Nervous system } \\
\hline Recovery agitation & $2(0.82,0.03-3.1)$ & $0(0.0,0.0-1.1)$ & $0.82(-1.0,2.3)$ \\
\hline Myoclonus & $0(0.0,0.0-1.2)$ & $0(0.0,0.0-1.1)$ & $0.01(-1.7,1.9)$ \\
\hline \multicolumn{4}{|l|}{ Gastrointestinal } \\
\hline Nausea & $0(0.0,0.0-1.2)$ & $1(0.36,0.001-2.2)$ & $-0.4(-2.3,1.6)$ \\
\hline Emesis & $1(0.41,0.001-2.5)$ & $0(0.0,0.0-1.1)$ & $0.4(-1.6,2.3)$ \\
\hline \multicolumn{4}{|l|}{ Other } \\
\hline Use of reversal agent & $4(1.6,0.05-4.3)$ & $5(1.8,0.2-3.3)$ & $-0.19(-2.9,2.9)$ \\
\hline Prolonged recovery time & $3(1.2,0.03-3.7)$ & $1(0.36,0.001-2.2)$ & $0.83(-1.7,3.5)$ \\
\hline Unplanned admission & $0(0.0,0.0-1.2)$ & $0(0.0,0.0-1.1)$ & $0.01(-1.7,1.9)$ \\
\hline Chest compressions & $0(0.0,0.0-1.2)$ & $0(0.0,0.0-1.1)$ & $0.01(-1.7,1.9)$ \\
\hline Death & $0(0.0,0.0-1.2)$ & $0(0.0,0.0-1.1)$ & $0.01(-1.7,1.9$ \\
\hline
\end{tabular}




\begin{tabular}{|c|c|c|c|c|c|c|c|c|c|}
\hline $\begin{array}{l}\text { Age (yr), } \\
\text { Sex, IDU/ } \\
\text { non-IDU } \\
\text { status }\end{array}$ & $\begin{array}{l}\text { Patient } \\
\text { opioid use }\end{array}$ & $\begin{array}{l}\text { Abscess } \\
\text { location }\end{array}$ & $\begin{array}{l}\text { Weight } \\
(\mathrm{kg})\end{array}$ & $\begin{array}{l}\text { Pre- } \\
\text { PSA } \\
\text { med }\end{array}$ & Med1 & Med 2 & $\begin{array}{l}\text { Description of } \\
\text { adverse event } \\
\text { with vital signs }\end{array}$ & Intervention & $\begin{array}{l}\text { Recovery } \\
\text { (min) }\end{array}$ \\
\hline \multicolumn{10}{|l|}{ Respiratory } \\
\hline $35, M, I D U^{*}$ & No & Perianal & 136 & None & $\mathrm{F} 150 \mu \mathrm{g}$ & M $2 \mathrm{mg}$ & $\begin{array}{l}\text { Hypoxia: initial } \mathrm{O}_{2} \\
97 \% \text {, nadir } \mathrm{O}_{2} 79 \%\end{array}$ & $\begin{array}{l}\text { Naloxone } 0.4 \mathrm{mg} \\
\text { BVM } \times 1 \mathrm{~min}\end{array}$ & 36 \\
\hline 36, M, IDU & No & Arm & 70 & None & P 330 mg & $\mathrm{F} 150 \mu \mathrm{g}$ & $\begin{array}{l}\text { Hypoxia, initial } \\
\mathrm{O}_{2} 100 \% \text {, nadir } \\
\mathrm{O}_{2} 75 \%\end{array}$ & $\mathrm{BVM} \times 2 \min$ & 33 \\
\hline $41, \mathrm{M}, \mathrm{IDU} \mathrm{H}^{*}$ & Yes & Leg & 70 & Mor 5 & $\mathrm{~F} 350 \mu \mathrm{g}$ & M 5 mg & $\begin{array}{l}\text { Apnea: initial } \mathrm{O}_{2} \\
99 \% \text {, nadir } \\
\mathrm{O}_{2} 93 \%\end{array}$ & $\begin{array}{l}\text { Flumazenil } \\
0.1 \mathrm{mg}\end{array}$ & 26 \\
\hline $27, \mathrm{M}, \mathrm{IDU}$ * & Yes & Hand & 77 & Mor 5 & $\mathrm{~F} 500 \mu \mathrm{g}$ & M 4 mg & $\begin{array}{l}\text { Apnea: initial } \\
\mathrm{O}_{2} 99 \% \\
\text { nadir } \mathrm{O}_{2}, 90 \%\end{array}$ & $\begin{array}{l}\text { Flumazenil } \\
0.1 \mathrm{mg}\end{array}$ & 35 \\
\hline 31, F, IDU* & Yes & Arm & 68 & None & P 50 mg & $\mathrm{F} 50 \mu \mathrm{g}$ & $\begin{array}{l}\text { Apnea: initial } \\
\mathrm{O}_{2} 98 \% \text {, } \\
\text { nadir } \mathrm{O}_{2} 91 \%\end{array}$ & $\begin{array}{l}\text { Naloxone } \\
0.2 \mathrm{mg}\end{array}$ & 73 \\
\hline 51, M, non-IDU & No & Hand & 89 & None & P 200 mg & $\mathrm{F} 175 \mu \mathrm{g}$ & $\begin{array}{l}\text { Hypoxia: initial } \\
\mathrm{O}_{2} 98 \%, \\
\text { nadir } \mathrm{O}_{2} 84 \%,\end{array}$ & $\begin{array}{l}\text { Oral airway } \\
\times 2 \text { min }\end{array}$ & 45 \\
\hline 35, M, non-IDU* & No & Buttock & 136 & None & $\mathrm{F} 200 \mu \mathrm{g}$ & M 2 mg & $\begin{array}{l}\text { Hypoxia: initial } \\
\mathrm{O}_{2} 98 \% \text {, } \\
\text { nadir } \mathrm{O}_{2} 78 \%\end{array}$ & $\begin{array}{l}\text { Naloxone } \\
0.4 \mathrm{mg} \text {, oral } \\
\text { airway } \times 1 \mathrm{~min}\end{array}$ & 47 \\
\hline $64, F$, non-IDU* & No & Perianal & 84 & None & $\mathrm{F} 250 \mu \mathrm{g}$ & M 3 mg & $\begin{array}{l}\text { Hypoxia: initial } \\
\mathrm{O}_{2} 98 \% \\
\text { nadir } \mathrm{O}_{2} 75 \%\end{array}$ & $\begin{array}{l}\text { Naloxone } \\
0.4 \mathrm{mg}, \mathrm{BVM} \times \\
2 \mathrm{~min}\end{array}$ & 4 \\
\hline $24, \mathrm{M}$, non-IDU* & No & Buttock & 70 & None & $\mathrm{F} 250 \mu \mathrm{g}$ & M $5 \mathrm{mg}$ & $\begin{array}{l}\text { Apnea: initial } \\
\mathrm{O}_{2} 99 \% \\
\text { nadir } \mathrm{O}_{2}, 80 \%\end{array}$ & $\begin{array}{l}\text { Naloxone } \\
0.2 \mathrm{mg}\end{array}$ & 20 \\
\hline 29, M, non-IDU* & No & Leg & 89 & None & F $100 \mu \mathrm{g}$ & M 4 mg & $\begin{array}{l}\text { Apnea: initial } \\
\mathrm{O}_{2} 99 \% \\
\text { nadir } \mathrm{O}_{2}, 91 \%\end{array}$ & $\begin{array}{l}\text { Naloxone } \\
0.2 \mathrm{mg}\end{array}$ & 12 \\
\hline 47, M, non-IDU* & No & Abdomen & 84 & None & $\mathrm{F} 350 \mu \mathrm{g}$ & M 6 mg & $\begin{array}{l}\text { Apnea: initial } \\
\mathrm{O}_{2} 98 \% \text {, nadir } \\
\mathrm{O}_{2} 90 \%\end{array}$ & $\begin{array}{l}\text { Naloxone } \\
0.4 \mathrm{mg}\end{array}$ & 9 \\
\hline 49, M, non-IDU & No & Leg & 141 & None & $\mathrm{F} 250 \mu \mathrm{g}$ & M 3 mg & $\begin{array}{l}\text { Partial airway } \\
\text { obstruction }\end{array}$ & $\begin{array}{l}\text { Airway } \\
\text { positioning }\end{array}$ & 14 \\
\hline 67, M, non-IDU & No & Buttock & 120 & Mor 5 & P 100 mg & $\mathrm{F} 250 \mu \mathrm{g}$ & $\begin{array}{l}\text { Partial airway } \\
\text { obstruction }\end{array}$ & $\begin{array}{l}\text { Airway } \\
\text { positioning }\end{array}$ & 25 \\
\hline \multicolumn{10}{|l|}{ Cardiovascular } \\
\hline $48, M, I D U$ & No & Arm & 70 & None & P 240 mg & F $75 \mu \mathrm{g}$ & $\begin{array}{l}\text { Hypotension: initial } \\
\text { BP } 105 / 60 \text { mm Hg, } \\
\text { nadir BP } \\
85 / 50 \text { mm Hg }\end{array}$ & NS $1 \mathrm{~L}$ bolus & 28 \\
\hline 31, F, IDU & Yes & Leg & 55 & None & $\mathrm{F} 500 \mu \mathrm{g}$ & M 4 & $\begin{array}{l}\text { Hypotension: initial } \\
\text { BP 104/76 mm Hg, } \\
\text { nadir BP } \\
78 / 55 \text { mm Hg }\end{array}$ & $\begin{array}{l}\text { NS } 500 \mathrm{cc} \\
\text { bolus }\end{array}$ & 104 \\
\hline 36, F, IDU & Yes & Buttock & 56 & Mor 3 & P 340 mg & F $75 \mu \mathrm{g}$ & $\begin{array}{l}\text { Hypotension: initial } \\
\text { BP 92/58 mm Hg, } \\
\text { nadir BP } \\
70 / 50 \text { mm Hg }\end{array}$ & NS $1 \mathrm{~L}$ bolus & 158 \\
\hline \multicolumn{10}{|l|}{ Nervous system } \\
\hline $31, F, I D U$ & No & Arm & 68 & None & K 50 mg & P $50 \mathrm{mg}$ & $\begin{array}{l}\text { Recovery agitation; } \\
\text { unpleasant reaction }\end{array}$ & M 5 mg IV & 73 \\
\hline $58, F, I D U$ & No & Leg & 86 & None & K 40 mg & P $40 \mathrm{mg}$ & $\begin{array}{l}\text { Recovery agitation; } \\
\text { yelling }\end{array}$ & M 2 mg IV & 35 \\
\hline
\end{tabular}




\begin{tabular}{|c|c|c|c|c|c|c|c|c|c|}
\hline $\begin{array}{l}\text { Age (yr), } \\
\text { Sex, IDU/ } \\
\text { non-IDU } \\
\text { status }\end{array}$ & $\begin{array}{l}\text { Patient } \\
\text { opioid use }\end{array}$ & $\begin{array}{l}\text { Abscess } \\
\text { location }\end{array}$ & $\begin{array}{l}\text { Weight } \\
(\mathrm{kg})\end{array}$ & $\begin{array}{l}\text { Pre- } \\
\text { PSA } \\
\text { med }\end{array}$ & Med1 & Med 2 & $\begin{array}{l}\text { Description of } \\
\text { adverse event } \\
\text { with vital signs }\end{array}$ & Intervention & $\begin{array}{c}\text { Recovery } \\
\text { (min) }\end{array}$ \\
\hline \multicolumn{10}{|l|}{ Other } \\
\hline $26, F, I D U$ & Yes & Arm & 43 & None & K 40 mg & P $60 \mathrm{mg}$ & Emesis & $\begin{array}{l}\text { Metoclopramide } \\
10 \mathrm{mg} \mathrm{IV}\end{array}$ & 50 \\
\hline $\begin{array}{l}\text { 33, F, } \\
\text { non-IDU }\end{array}$ & No & Leg & 45 & None & $\mathrm{F} 100 \mu \mathrm{g}$ & M 2 mg & Nausea & $\begin{array}{l}\text { Dimenhydrinate } \\
25 \mathrm{mg} \text { IV }\end{array}$ & 30 \\
\hline $27, F, I D U$ & Yes & Arm & 59 & Mor 5 & $\mathrm{~F} 50 \mu \mathrm{g}$ & M $5 \mathrm{mg}$ & Recovery time $>3 \mathrm{~h}$ & None & 219 \\
\hline 26, M, IDU & No & Arm & 71 & None & K 40 mg & P $20 \mathrm{mg}$ & Recovery time $>3 \mathrm{~h}$ & None & 255 \\
\hline $26, F, I D U$ & Yes & Arm & 68 & None & P 100 mg & $\mathrm{F} 50 \mu \mathrm{g}$ & Recovery time $>3 \mathrm{~h}$ & None & 205 \\
\hline $\begin{array}{l}\text { 51, M, } \\
\text { non-IDU }\end{array}$ & No & Arm & 70 & None & $\mathrm{F} 250 \mu \mathrm{g}$ & M 4 mg & Recovery time $>3 \mathrm{~h}$ & None & 190 \\
\hline
\end{tabular}

$\mathrm{BP}=$ blood pressure; $\mathrm{BVM}=$ bag-valve mask; $\mathrm{F}=$ fentanyl; $\mathrm{IDU}=$ injection drug user; $\mathrm{K}=$ ketamine; $\mathrm{M}=$ midazolam; Mor $=$ morphine; $\mathrm{NS}=$ normal saline; $\mathrm{O}_{2}=$ oxygen saturation; $\mathrm{P}=$ propofol; Pre-PSA med = parenteral opioid given within 30 minutes of starting sedation; Med 1, Med $2=$ medications administered during procedural sedation and analgesia

*Each patient had two adverse events.

coadministered with either propofol or midazolam. AEs in patients receiving ketamine/propofol or propofol alone were not strongly influenced by dose. However, the small number of AEs and nonrandom assignment preclude drawing firm conclusions regarding the relative safety of the sedating regimens.

Rapid recovery promotes patient safety and reduces ED resource use. The 18-minute IDU recovery time was 6 minutes longer than the non-IDU recovery period. This could be due to the greater amounts of sedative and analgesic medications received by the IDU group or reflect the pre-existing acute and chronic health problems observed in that group. Despite eventually being discharged from the ED, patients experiencing AEs had substantially longer median recovery times - 59 minutes in the IDU group (versus 18 minutes for the overall IDU group) and 20 minutes (versus 12 minutes in the non-IDU group)—again suggesting a relationship with excessive dosing.

Nine patients were administered reversal agents. Use of flumazenil or naloxone, although causing a patient to "recover" quickly from PSA, may also precipitate rapid opioid or benzodiazepine withdrawal. Many patients depend on these medications, and use of reversal agents that could lead to agitation, seizures, or emesis should be carefully considered.

\section{LIMITATIONS}

Although the physicians, nurses, and respiratory therapists who recorded the data all underwent PSA and documentation training, there was no independent data quality control during the study period. The physicians and nurses who completed data sheets were aware of IDU status, as were the reviewers, and AEs may have been misclassified. The IDU status may be problematic: a patient who used injection drugs once in the past month may be less prone to AEs than someone who inhaled cocaine on a daily basis.

Patient self-administration of drugs, including opioids, prior to attending the ED was not recorded. Weightadjusted dosing could be estimated only for the $9 \%$ of patients who did not have weights recorded. Willingness to administer PSA, incision and drainage, and the choice and dosing of sedatives and analgesics was entirely at the discretion of the treating physician. Variability in the selection, dosing, and timing of medications could reflect that physician's experience and/or bias in this patient population. Adjuvant use of local or regional anesthesia was individualized but not consistently recorded.

Some AEs, especially minor airway readjustments, may not have been documented. Capnography was not mandated in our region until after the study was completed. Measuring carbon dioxide partial pressure could have increased both interventions and reported AEs. ${ }^{25-27}$ Sedation effectiveness and patient and staff satisfaction scores were not obtained.

\section{CONCLUSION}

For ED patients undergoing PSA for incision and drainage, IDUs had a rate of AEs similar to that of 

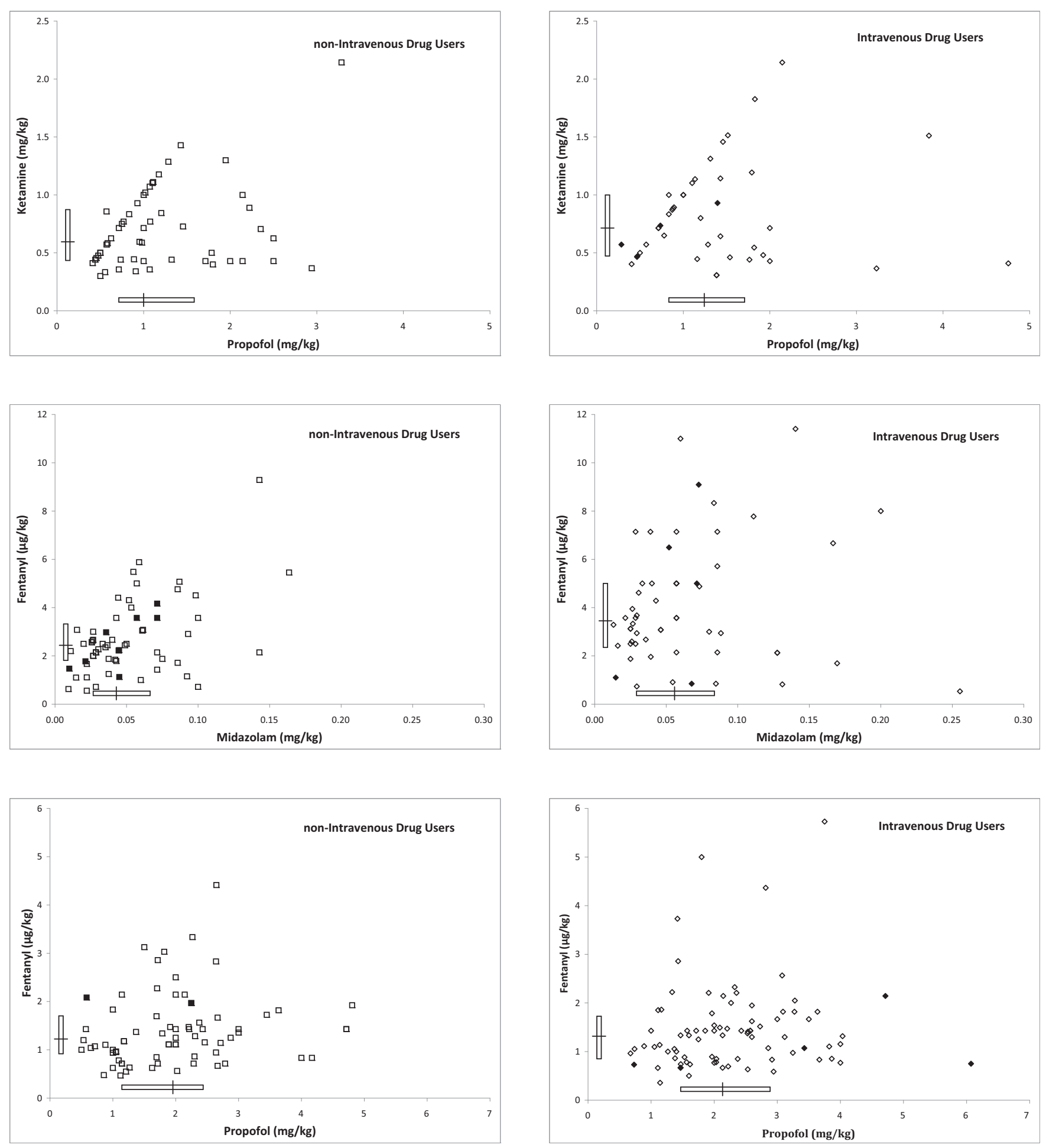

Figure 1. Adverse events by sedating regimen for injection drug users and non-injection drug users. Cumulative drug dose per patient weight administered during the entire procedure is shown. Filled symbols represent the patients experiencing an adverse event; open symbols represent the rest. The rectangle along each axis spans the 25th to 75th percentile, whereas the bar indicates the median. A patient weight of $70 \mathrm{~kg}$ was used for the $9 \%$ of patients in whom weight was not recorded. 
Procedural sedation for injection drug users

\section{Table 6. Secondary outcomes}

\begin{tabular}{|c|c|c|c|}
\hline Category & $\operatorname{IDU}(n=244)$ & Non-IDU $(n=281)$ & $\%$ difference $(95 \% \mathrm{Cl})^{*}$ \\
\hline \multicolumn{4}{|l|}{ Times (median, IQR) } \\
\hline Recovery time (min) & $18(10,36)$ & $12(7,19)$ & $6(2-9)$ \\
\hline Sedation time (min) & $25(17,45)$ & $19(14,25)$ & $6(5-10)$ \\
\hline \multicolumn{4}{|l|}{ ED disposition } \\
\hline Admitted to hospital, $n(\%)$ & $20(8.2)$ & $1(0.4)$ & $7.8(4.3-12.2)$ \\
\hline
\end{tabular}

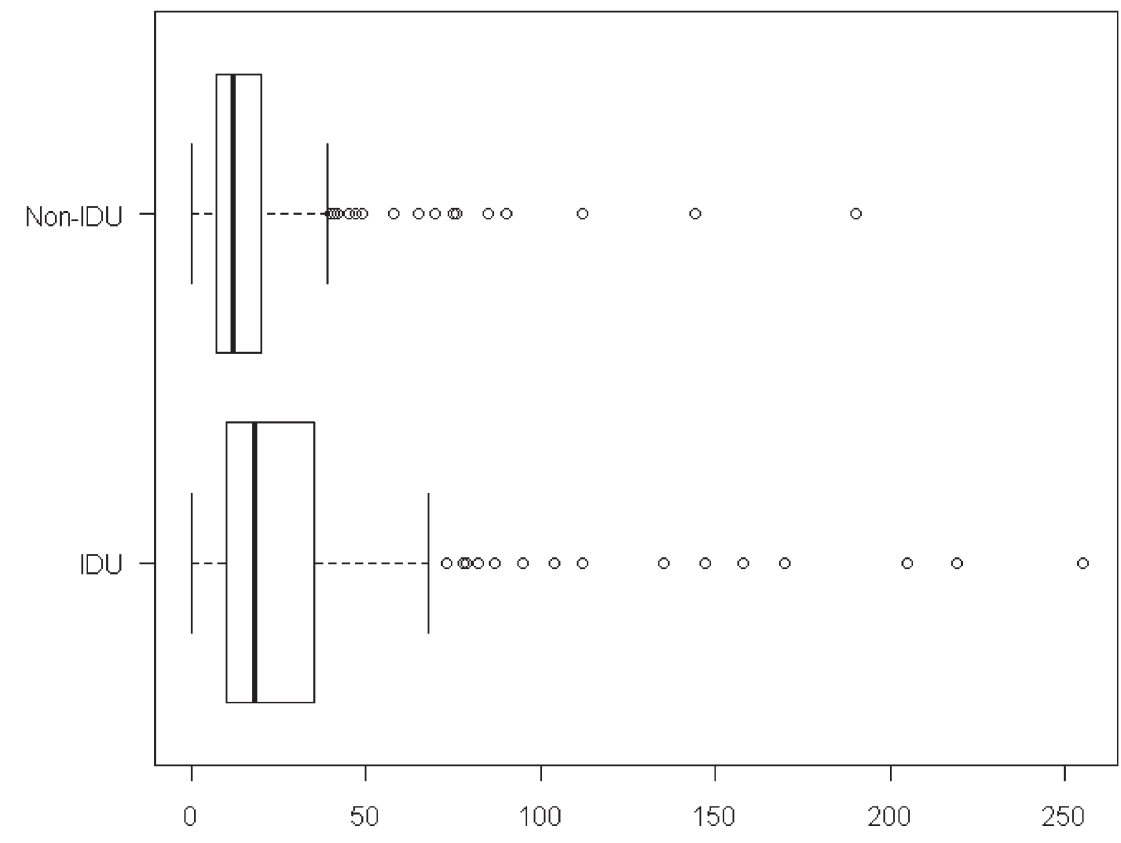

A

Recovery Time (in minutes)

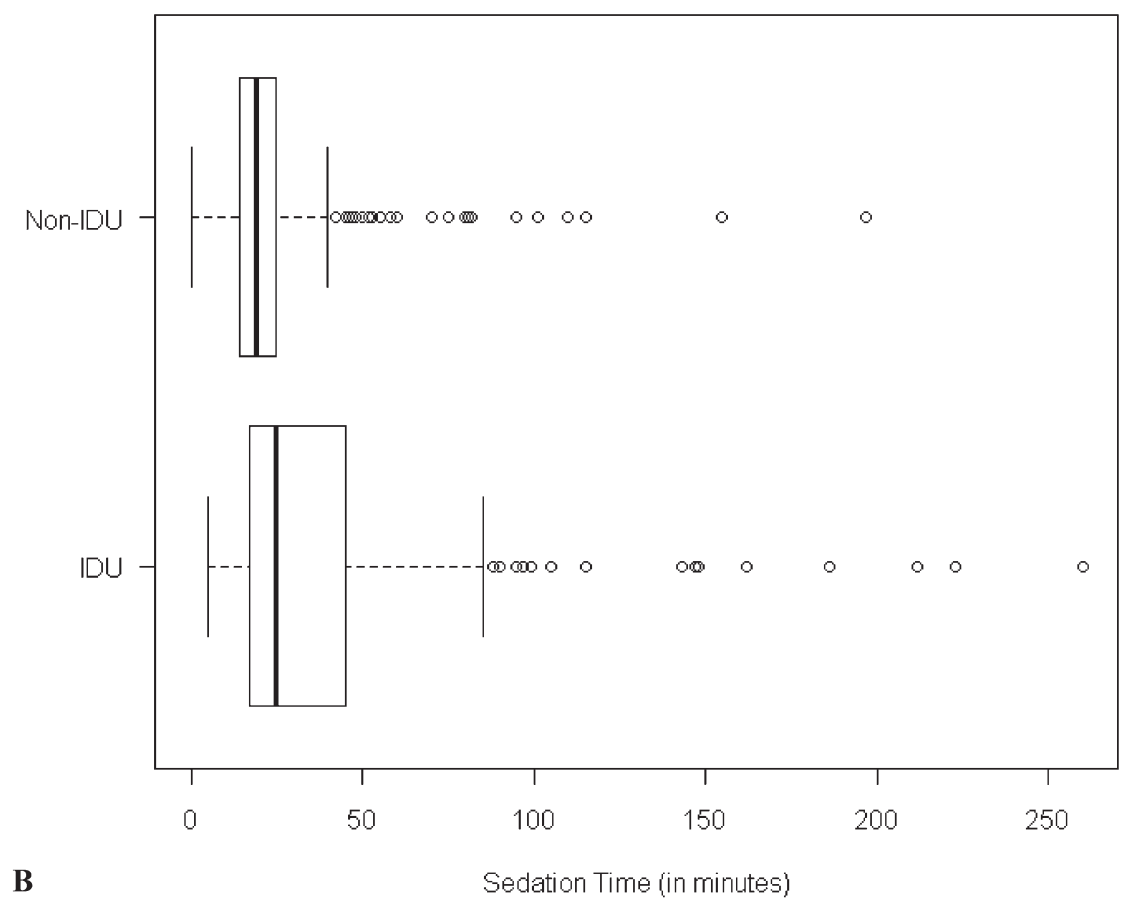

Figure 2. $A$, Recovery time (time from last administration of sedative/analgesic drug until physiologic recovery) for injection drug users (IDUs) and non-IDUs. $B$, Sedation time (time from first administration of sedative/analgesic drug to physiologic recovery) for IDUs and non-IDUs. 
non-IDUs but longer recovery times. When performed by experienced physicians, PSA may be as safe for this group of patients as for patients who do not use injection drugs.

Competing interests: None declared.

\section{REFERENCES}

1. World drug report. Vol 1. Analysis. New York: United Nations Office on Drugs and Crime; 2004.

2. Palepu A, Tyndall MW, Leon H, et al. Hospital utilization and costs in a cohort of injection drug users. CMA7 2001; 165:415-20.

3. Gordon RJ, Lowy FD. Bacterial infections in drug users. $N$ Engl 7 Med 2005;353:1945-54, doi:10.1056/NEJMra042823.

4. Kreek MJ. Cocaine, dopamine, and the endogenous opioid system. I Addict Dis 1996;15:73-96, doi:10.1300/ J069v15n04_05.

5. Silverman SM. Opioid induced hyperalgesia: clinical implications for the pain practitioner. Pain Physician 2009;12:67984.

6. Hay JL, White JM, Bochner F, et al. Hyperalgesia in opioidmanaged and opioid-dependent patients. 7 Pain 2009;10: 316-22, doi:10.1016/j.jpain.2008.10.003.

7. May JA, White HC, Leonard-White A, et al. The patient recovering from alcohol or drug addiction: special issues for the anesthesiologist. Anesth Analg 2001;92:1601-8, doi:10.1097/00000539-200106000-00050.

8. Kuczkowski KM. Anesthetic implications of drug abuse in pregnancy. 7 Clin Anesth 2003;15:382-94, doi:10.1016/ S0952-8180(03)00056-4.

9. Constantino TG, Parikh AK, Satz WA, et al. Ultrasonograph-guided peripheral intravenous access versus traditional approaches in patients with difficult intravenous access. Ann Emerg Med 2005;46:456-61, doi:10.1016/j.annemergmed.2004.12.026.

10. Mehta V, Langford RM. Acute pain management for opioid dependent patients. Anaesthesia 2006;61:269-76, doi:10.1111/ j.1365-2044.2005.04503.x.

11. Richebe P, Beaulieu P. Perioperative pain management in the patient treated with opioids: continuing professional development. Can 7 Anaesth 2009;56:969-81, doi:10.1007/ s12630-009-9202-y.

12. Scheuermeyer FX, Grafstein E, Stenstrom R, et al. Thirtyday outcomes of emergency department patients undergoing electrical cardioversion for atrial fibrillation or flutter. Acad Emerg Med 2010;17:408-15, doi:10.1111/j.1553-2712. 2010.00697.x.

13. Green SM, Krauss B. Procedural sedation terminology: moving beyond "conscious sedation." Ann Emerg Med 2002; 39:433-5, doi:10.1067/mem.2002.122770.
14. Palepu A, Tyndall MW, Leon H, et al. Hospital utilization and costs in a cohort of injection drug users. CMAJ 2001; $165: 415-20$.

15. Wood E, Tyndall MW, Spittal PM, et al. Unsafe injection practices in a cohort of injection drug users in Vancouver: could safer injecting rooms help? CMAJ 2001;165:405-10.

16. Kerr T, Stoltz J-A, Tyndall M, et al. Impact of a medically supervised safer injection facility on community drug use patterns: a before and after study. BM7 2006;332:220-2, doi:10.1136/bmj.332.7535.220.

17. Mensch BS, Kandel DB. Underreporting of substance use in a national longitudinal youth cohort, individual and interviewer effects. Public Opin Q 1988;52:100-24, doi:10.1086/ 269084.

18. Aldrete JA. The post-anesthesia recovery score revisited. 7 Clin Anesth 1995;7:89-91, doi:10.1016/0952-8180(94)00001-K.

19. Worster A, Bledsoe RD, Cleve P, et al. Reassessing the methods of medical record review studies. Ann Emerg Med 2005;45:448-51, doi:10.1016/j.annemergmed.2004.11.021.

20. Gilbert EH, Lowenstein SR, Kozoil-McLain J, et al. Chart reviews in emergency medicine research: where are the methods? Ann Emerg Med 1996;27:305-8, doi:10.1016/ S0196-0644(96)70264-0.

21. Willman EV, Andolfatto G. A prospective evaluation of "ketofol" (ketamine/propofol combination) for procedural sedation and analgesia in the emergency department. Ann Emerg Med 2007;49:23-30, doi:10.1016/j.annemergmed. 2006.08.002.

22. Andolfatto G, Willman EV. A prospective case series of single-syringe ketamine-propofol (ketofol) for emergency department procedural sedation and analgesia in adults. Acad Emerg Med 2011;18:237-45, doi:10.1111/j.1553-2712.2011. 01010.x.

23. Andolfatto G, Willman EV. A prospective case series of pediatric procedural sedation and analgesia in the emergency department using single-syringe ketamine-propofol combination (ketofol). Acad Emerg Med 2010;17:194-201, doi:10.1111/ j.1553-2712.2009.00646.x.

24. Murphy LJ. Hypertension and pulmonary edema associated with ketamine administration in a patient with a history of substance abuse. Can 7 Anaesth 1993;40:160-4, doi:10.1007/ BF03011314.

25. Deitch K, Miner J, Chudnofsky CR, et al. Does end tidal CO2 monitoring during emergency department procedural sedation and analgesia with propofol decrease the incidence of hypoxic events? A randomized, controlled trial. Ann Emerg Med 2010; 55:258-64, doi:10.1016/j.annemergmed.2009.07.030.

26. Miner JR, Heegaard W, Plummer D. End-tidal carbon dioxide monitoring during procedural sedation. Acad Emerg Med 2002;9:275-80, doi:10.1111/j.1553-2712.2002. tb01318.x.

27. Burton JH, Harrah JD, Germann CA, et al. Does end-tidal carbon dioxide monitoring detect respiratory events prior to current sedation monitoring practices? Acad Emerg Med 2006;13:500-4, doi:10.1111/j.1553-2712.2006.tb00999.x. 